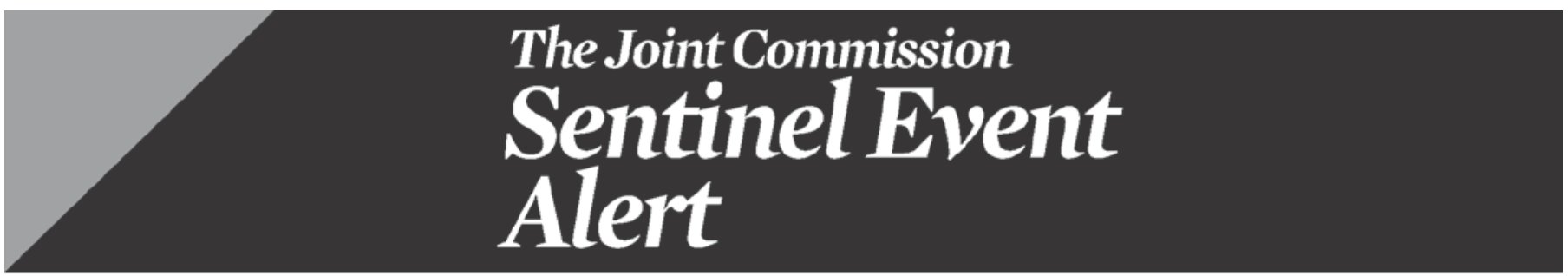

A complimentary publication of The Joint Commission

\section{Safe use of opioids in hospitals}

While opioid use is generally safe for most patients, opioid analgesics may be associated with adverse effects, ${ }^{1,2,3}$ the most serious effect being respiratory depression, which is generally preceded by sedation. ${ }^{4,5,6}$ Other common adverse effects associated with opioid therapy include dizziness, nausea, vomiting, constipation, sedation, delirium, hallucinations, falls, hypotension, and aspiration pneumonia. ${ }^{4,7}$ Adverse events can occur with the use of any opioid; among these are fentanyl, hydrocodone, hydromorphone, methadone, morphine, oxycodone, and sufentanil. While there are numerous problems associated with opioid use, including underprescribing, overprescribing, tolerance, dependence, and drug abuse, this Alert will focus on the safe use of opioids that are prescribed and administered within the inpatient hospital setting. The Joint Commission recognizes that the emergency department presents unique challenges that should also be addressed by the hospital, but may not be directly addressed in this Alert. This Alert will provide a number of actions that can be taken to avoid the unintended consequences of opioid use among hospital inpatients.

Opioid analgesics rank among the drugs most frequently associated with adverse drug events. The literature provides numerous studies of the adverse events associated with opioids. One study found that most adverse drug events were due to drug-drug interactions, most commonly involving opioids, benzodiazepines, or cardiac medications. ${ }^{8}$ In addition, a British study of 3,695 inpatient adverse drug reactions found that 16 percent were attributable to opioids, making opioids one of the most frequently implicated drugs in adverse reactions. ${ }^{7}$ The incidence of respiratory depression among post-operative patients is reported to average about 0.5 percent. Some of the causes for adverse events associated with opioid use are: - Lack of knowledge about potency differences among opioids.

- Improper prescribing and administration of multiple opioids and modalities of opioid administration (i.e., oral, parenteral and transdermal patches).

- Inadequate monitoring of patients on opioids. ${ }^{9,10}$

Of the opioid-related adverse drug events - including deaths - that occurred in hospitals and were reported to The Joint Commission's Sentinel Event database (2004-2011), 47 percent were wrong dose medication errors, 29 percent were related to improper monitoring of the patient, and 11 percent were related to other factors, including excessive dosing, medication interactions and adverse drug reactions. ${ }^{*}$ These reports underscore the need for the judicious and safe prescribing and administration of opioids, and the need for appropriate monitoring of patients. When opioids are administered, the potential for opioid-induced respiratory depression should always be considered because:

- The risk may be greater with higher opioid doses

- The occurrence may actually be higher than reported

- There is a higher incidence observed in clinical trials ${ }^{11}$

- Various patients are at higher risk (see below), including patients with sleep apnea, patients who are morbidly obese, who are very young, who are elderly, who are very ill, and who concurrently receive other drugs that are central nervous system and respiratory depressants (e.g., anxiolytics, sedatives). ${ }^{5,11,12}$

\footnotetext{
* The reporting of most sentinel events to The Joint Commission is voluntary and represents only a small proportion of actual events. Therefore these data are not an epidemiologic data set and no conclusions should be drawn about the actual relative frequency of events or trends in events over time.
} 
The need for assessing and managing pain to help avoid accidental opioid overdose

The safe use of opioids in hospital settings relies on an accurate pain assessment and then applying appropriate pain management techniques. ${ }^{1,2,3}$ The Joint Commission's pain management standards have increased awareness of the importance of safe and effective pain management. ${ }^{13}$ Instituted in 2001, these standards have made a significant difference in appropriate pain management. Before the standards, there were continuing reports of inadequate pain control for postoperative and traumatic pain, cancer pain, and many other acute and chronic pain challenges. ${ }^{13}$ In addition, compliance with the standards leads to better clinical outcomes, such as improved wound healing, and helps to prevent untoward consequences of inadequate pain relief, such as impaired immune response. ${ }^{14}$ The importance of both assessing and managing pain is critical to patients who otherwise would suffer debilitating pain. Notwithstanding the need for appropriate pain management, organizations should make staff aware of the following factors and activities that can help avoid accidental opioid overuse:

- Screen patients for respiratory depression risk factors (see sidebar). ${ }^{5,11,12}$

- Assess the patient's previous history of analgesic use or abuse, duration and possible side effects to identify potential opioid tolerance or intolerance.

- Conduct a full body skin assessment of patients prior to administering a new opioid to rule out the possibility that the patient has an applied fentanyl patch or implanted drug delivery system or infusion pump.

- Use an individualized, multimodal treatment plan to manage pain. ${ }^{6,15} \mathrm{~A}$ multimodal approach combines strategies such as psychosocial support, coordination of care, the promotion of healthful behaviors, nonpharmacologic approaches, and nonopioid pain medications. ${ }^{15,16,17}$ Upon assessment, the best approach may be to start with a non-narcotic. The Joint Commission recognizes that not all pain can be eliminated; therefore, our standards provide for goal-related therapy. For example, a patient may define a pain level that is tolerable and acceptable on the pain scale (e.g., level three on a 10-point scale).

- Take extra precautions with patients who are new to opioids or who are being restarted on opioids. These precautions should include starting the patient with a short-term trial ${ }^{15}$ of carefully titrated opioids at the lowest effective dose to achieve satisfactory pain control. ${ }^{18}$ Sufficient time should be allowed to assess the
Characteristics of patients who are at

higher risk for oversedation and

respiratory depression

- Sleep apnea or sleep disorder diagnosis ${ }^{5}$

- Morbid obesity with high risk of sleep apnea ${ }^{5}$

- $\quad$ Snoring ${ }^{5,6}$

- Older age; risk is

o 2.8 times higher for individuals aged $61-70$

o $\quad 5.4$ times higher for age 71-80

o 8.7 times higher for those over age $80^{5}$

- $\quad$ No recent opioid use $e^{6,21}$

- Post-surgery, particularly if upper abdominal or thoracic surgery

- Increased opioid dose requirement ${ }^{6}$ or opioid habituation

- Longer length of time receiving general anesthesia during surgery ${ }^{5,23}$

- Receiving other sedating drugs, such as benzodiazepines, antihistamines, diphenhydramine, sedatives, or other central nervous system depressants

- Preexisting pulmonary or cardiac disease or dysfunction or major organ failure

- Thoracic or other surgical incisions that may impair breathing

- Smoker

patient's response to an initial dose before increasing the dosage or prescribing opioids for long-term use.

- Consult a pharmacist or pain management expert (when available) when converting from one opioid to another, or changing the route of administration (from oral to IV or transdermal). Consider that the patient may be less tolerant of the new drug (incomplete cross tolerance) or that the new drug may be more potent. Note: While there are numerous dose conversion scales and other tools available, each organization should determine the tool(s) that will be used and assess staff's understanding of the selected tool(s). Sentinel events have been reported to The Joint Commission related to misuse or misunderstanding of these tools.

- Avoid rapid dose escalation of opioid analgesia above routine dose levels in opioidtolerant patients.

- Take extra precautions when transferring patients between care units and facilities, and 
when discharging patients to their home. Consider that drug levels may reach peak concentrations during transport.

- $\quad$ Avoid using opioids to meet an arbitrary pain rating or a planned discharge date. Dosing should be based on the individual patient's need and condition.

The Anesthesia Patient Safety Foundation (APSF) and the Institute for Safe Medication Practices (ISMP) and other organizations recommend continuous monitoring of oxygenation and/or ventilation of patients receiving opioids postoperatively.

Actions suggested by The Joint Commission Hospitals can take the following evidence-based actions to help avoid adverse events associated with the use of opioids.

\section{Effective processes}

1. Create and implement policies and procedures for the ongoing clinical monitoring of patients receiving opioid therapy by performing serial assessments of the quality and adequacy of respiration and the depth of sedation. ${ }^{6}$ The organization will need to determine how often the assessments should take place and define the period of time that is appropriate to adequately observe trends. ${ }^{6}$ Monitoring should be individualized according to the patient's response. ${ }^{19}$ The assessments are particularly important when the dose has been increased or another type of opioid is administered. In addition to monitoring respiration and sedation, pulse oximetry can be used to monitor oxygenation, and capnography can be used to monitor ventilation. Staff should be educated not to rely on pulse oximetry alone because pulse oximetry can suggest adequate oxygen saturation in patients who are actively experiencing respiratory depression, especially when supplemental oxygen is being used - thus the value of using capnography to monitor ventilation. ${ }^{6}$ When pulse oximetry or capnography is used, it should be used continuously rather than intermittently. ${ }^{6,19,24}$

2. Create and implement policies and procedures that allow for a second level review by a pain management specialist or pharmacist of pain management plans that include high-risk opioids, such as methadone, fentanyl, IV hydromorphone and meperidine. ${ }^{25,26}$

3. Create and implement policies and procedures for tracking and analyzing opioid-related incidents for quality improvement purposes. ${ }^{5}$
See relevant Joint Commission requirements: LD.04.01.07 element of performance 1, LD.04.04.05, PC.01.02.01, PC.01.02.03 EP 2 and 3, PI.01.01.01, PI.02.01.01, PI.03.01.01, MM.07.01.03

\section{Safe technology}

4. If available, use information technology to monitor prescribing of opioids.

- Build red flags or alerts into e-prescribing systems for all opioids. The red flags can be either for dosing limits or alerts, or for verifications.

- Separate sound-alike and look-alike opioids, and use tall man lettering and other techniques to reduce the risk of error.

- Use conversion support systems to calculate correct doses of opioids to help prevent problems with conversions from oral, IV and transdermal routes of administration.

- Use patient-controlled analgesia (PCA) to reduce the risk of oversedation. (See Sentinel Event Alert \#33, "Patient Controlled Analgesia by Proxy," for strategies for eliminating risk related to PCA by proxy.) The use of smart infusion pump technology with dosage error reduction software can add another layer of safety.

See relevant Joint Commission requirement: MM.01.01.03

\section{Appropriate education and training}

5. Advise clinicians who prescribe pain medications to use both pharmacologic and nonpharmacologic alternatives, including multi-modal adjuvant therapies (e.g., physical therapy, acupuncture, manipulation or massage, ice, music therapy). Non-narcotic analgesics, such as acetaminophen, nonsteroidal anti-inflammatory agents, antidepressants, anticonvulsants (e.g., gabapentin and pregabalin), and muscle relaxants (e.g., baclofen, tizanidine), can be used before prescribing an opioid. In addition, when used in combination with opioids, these non-narcotics may reduce the dose of opioids required to effectively manage pain. $^{27,28,29,30}$

6. Educate and assess the understanding of staff that care for patients receiving opioids about the potential effect of opioid therapy on sedation and respiratory depression, the continuum of consciousness, the difference between ventilation and oxygenation, and technological and clinical monitoring. Staff training should emphasize how to assess patients for adverse drug reactions, how to recognize advancing sedation, and the importance of making timely adjustments to the plan of care 
based on the patient's risk. ${ }^{5,6}$ For example, a comprehensive pain management program can help to educate clinicians, endorse best practices, and improve safety.

7. Educate and provide written instructions to patients who are on opioids (and to the patient's family or caregiver) about:

- The various generic and brand names, formulations, and routes of administration of opioids in order to prevent confusion and reduce the accidental duplication of opioid prescriptions;

- The principal risks and side effects of opioids, including the likelihood of constipation, and the risk of falls, nausea and vomiting;

- The impact of opioid therapy on psychomotor and cognitive function (which may affect driving and work safety);

- The potential for serious interactions with alcohol and other central nervous system depressants;

- The potential risks of tolerance, addiction, physical dependency, and withdrawal symptoms associated with opioid therapy. ${ }^{15}$

- The specific dangers as a result of the potentiating effects when opioids are used in combination, such as oral and transdermal (fentanyl patches).

- $\quad$ The safe and secure storage of opioid analgesics in the home.

When providing this information at discharge, also include phone numbers for a contact person to call with questions.

8. Assess the organization's need for training based on the analysis of reported adverse events, near misses and staff observations. This analysis may be helpful in identifying knowledge gaps and in developing improvement strategies to reduce recurrences.

See relevant Joint Commission requirements: HR.01.04.01 EP 4, HR.01.05.03, HR.01.06.01, MS.03.01.03 EP 2

\section{Effective tools}

9. Provide standardized tools that can be used to screen patients for risk factors associated with oversedation and respiratory depression. Among the available screening tools for patients in the acute care setting are the Pasero Opioid-Induced Sedation Scale (POSS) and the Richmond Agitation-Sedation Scale (RASS). Tools that can be used after discharge to help prevent opioid misuse include the Screener and Opioid Assessment for Patients with Pain (SOAPP and SOAPP-R), the Opioid Risk Tool (ORT), and the
Screening Instrument for Substance Abuse Potential (SISAP).

See relevant Joint Commission requirement: PC.01.02.07 EP 2

Contributing to this alert were Judith A. Paice, Ph.D., R.N., director of the Cancer Pain Program at Northwestern University's Feinberg School of Medicine, Chicago, III.; Debra B. Gordon, R.N., University of Wisconsin Hospital and Clinics, Madison, Wis.; Jose Contreras, M.D., Pain and Palliative Medicine, Hackensack University Medical Center, Hackensack, N.J.; and Donna Jarzyna, R.N., University Medical Center, Tucson, Ariz.

\section{Resources}

The Food and Drug Administration provides a "Blueprint for Prescriber Education for ExtendedRelease and Long-Acting Opioid Analgesics," which includes information about the specific characteristics of the ER/LA opioid analgesic products.

\footnotetext{
References

${ }^{1}$ Vila H Jr, Smith RA, Augustyniak MJ: The efficacy and safety of pain management before and after implementation of hospital-wide pain management standards: Is patient safety compromised by treatment based solely on numerical pain ratings? Anesthesia and Analgesia, 2005;101:474-80

2 Emergency department visits involving nonmedical use of selected prescription drugs - United States, 20042008. Morbidity and Mortality Weekly Report 2010, 59:705-709

${ }^{3}$ Office of Applied Studies, Substance Abuse and Mental Health Services Administration. Substance abuse treatment admissions involving abuse of pain relievers: 1998 and 2008, http://oas.samhsa.gov/2k10/230/230PainRelvr2k10.cfm (accessed October 28, 2011)

${ }^{4}$ McPherson ML: Strategies for the management of opioid-induced adverse effects. Advanced Studies in Pharmacy, 2008;5(2):52-57

${ }^{5}$ Jarzyna D, et al: American Society for Pain Management Nursing guidelines on monitoring for opioidinduced sedation and respiratory depression. Pain Management Nursing, 2011;12(3):118-145.e10

${ }^{6}$ Pasero C, M McCaffery: Pain assessment and pharmacologic management. Chapter 12 - Key Concepts in Analgesic Therapy, and Chapter 19 - Management of opioid-induced adverse effects. St. Louis, Mosby Elseveir, 2011

${ }^{7}$ Davies EC, et al: Adverse Drug Reactions in Hospital In-Patients: A Prospective Analysis of 3695 PatientEpisodes, PLos ONE, February 2009;4(2):e4439

${ }^{8}$ Wright $A$, et al: Preventability of adverse drug events involving multiple drugs using publicly available clinical decision support tools. American Journal of HealthSystem Pharmacy, 2012; 69:221-7

${ }_{9}$ U.S. Food and Drug Administration: Public Health Advisories (Drugs), Fentanyl Transdermal Patch, Important Information for the Safe Use of Fentanyl
} 
Transdermal System (Patch). December 21, 2007, http://www.fda.gov/Drugs/DrugSafety/PostmarketDrugSa fetylnformationforPatientsandProviders/DrugSafetylnform ationforHeathcareProfessionals/PublicHealthAdvisories/u cm051257.htm?utm campaign=Google2\&utm source=fd aSearch\&utm medium=website\&utm term=fentanyl\&utm content $=9$ (accessed May 21, 2012)

${ }^{10}$ U.S. Food and Drug Administration: FDA Reminds the Public about the Potential for Life-Threatening Harm from Accidental Exposure to Fentanyl Transdermal Systems ("Patches"). April 18, 2012,

http://www.fda.gov/Drugs/DrugSafety/ucm300747.htm?ut m_campaign=Google2\&utm_source $=$ fdaSearch\&utm_me dium=website\&utm_term $=2012$ fentanyl\&utm_content $=4$ (accessed May 21, 2012)

${ }^{11}$ Dahan A, et al: Incidence, reversal and prevention of opioid-induced respiratory depression. Anesthesiology, 2010;112:226-38

${ }^{12}$ American Geriatrics Society Panel on the Pharmacological Management of Persistent Pain in Older Persons: Pharmacologic Management of Persistent Pain in Older Persons. Journal of the American Geriatrics Society. 2009;57:1331-46

${ }^{13}$ Dahl JL, Gordon DB: Joint Commission pain standards: a progress report. APS Bulletin, 2002;12(6)

${ }^{14}$ Wells N, et al: Patient Safety and Quality: An Evidence-Based Handbook for Nurses. Chapter 17 Improving the quality of care through pain assessment and management. AHRQ Publication No. 08-0043, April 2008. Agency for Healthcare Research and Quality, Rockville, Md., http://www.ahrq.gov/qual/nurseshdbk/ ${ }^{15}$ Chou R, et al, on behalf of the American Pain Society American Academy of Pain Medicine Opioids Guidelines Panel: Clinical guidelines for the use of chronic opioid therapy in chronic noncancer pain. The Journal of Pain, 2009;10(2):113-130

${ }^{16}$ Harvard Health Publications: Painkillers fuel growth in drug addiction. Harvard Reviews of Health News, January 2011:4-5, http://harvardpartnersinternational.staywellsolutionsonlin e.com/69,M0111b (accessed October 28, 2011)

${ }^{17}$ Streltzer J, Johansen L: Prescription drug dependence and evolving beliefs about chronic pain management. American Journal of Psychiatry, 2006;163(4):594-597

${ }^{18}$ Pasero C, et al: Using continuous infusion with PCA. American Journal of Nursing, 1999;99(2)

${ }^{19}$ Overdyk FJ: Postoperative respiratory depression and opioids. Initiatives in Safe Patient Care, Saxe Healthcare Communications, 2009, http://initiativespatientsafety.org/Initiatives1\%20.pdf (accessed October 28, 2011)

${ }^{20}$ Cepeda MS, et al: Side effects of opioids during shortterm administration: effect of age, gender and race. Clinical Pharmacology and Therapeutics, 2003;74:102112

${ }^{21}$ Dunn KM, et al: Opioid prescriptions for chronic pain and overdose: a cohort study. Annals of Internal Medicine, 2010;152:85-92

${ }^{22}$ Hagle ME, et al: Respiratory depression in adult patients with intravenous patient-controlled analgesia. Orthopaedic Nursing, 2004;23(1)):18-27
${ }^{23}$ Ozdilekcan C, et al: Risk factors associated with postoperative pulmonary complications following oncological surgery. Tuberk Toraks, 2004;52(3):248-55 ${ }^{24}$ Stoelting RK, Weinger MB: Dangers of postoperative opioids - is there a cure? Anesthesia Patient Safety Foundation Newsletter, Summer 2009;24(2):25-26

${ }^{25}$ The Truax Group: Long-acting and extended-release opioid dangers. Patient Safety Tip of the Week, June 28, 2011,

http://patientsafetysolutions.com/docs/June 282011 Lo ng Acting and Extended Release Opioid Dangers.htm (accessed July 19, 2011)

${ }^{26}$ Institute For Safe Medication Practices: Ongoing, preventable fatal events with fentanyl transdermal patches are alarming! Medication Safety Alert, June 28, 2007,

http://www.ismp.org/newsletters/acutecare/articles/20070 628.asp (accessed October 28, 2011)

${ }^{27}$ Svenson JE, Meyer, TD: Effectiveness of nonnarcotic protocol for the treatment of acute exacerbations of chronic nonmalignant pain. The American Journal of Emergency Medicine, 2007;25:445-449

${ }^{28}$ Schug SA, Manopas A: Update on the role of nonopioids for postoperative pain treatment. Best Practice \& Research Clinical Anaesthesiology, 2007;21(1):15-30

${ }^{29}$ Munir MA, et al: Nonopioid analgesics. Anesthesiology Clinics, 2007;25:761-774

${ }^{30}$ White PF: The changing role of non-opioid analgesic techniques in the management of postoperative pain. Anesthesia \& Analgesia, 2005;101:S5-S22

\section{Patient Safety Advisory Group}

The Patient Safety Advisory Group informs The Joint Commission on patient safety issues and, with other sources, advises on topics and content for Sentinel Event Alert. Members: James P. Bagian, M.D., P.E. (chair); Michael Cohen, R.Ph., M.S., Sc.D. (vice chair); Jane H. Barnsteiner, R.N., Ph.D., FAAN; Jim B. Battles, Ph.D.; William H. Beeson, M.D.; Patrick J. Brennan, M.D.; Martin H. Diamond, FACHE; Cindy Dougherty, R.N., CPHQ; Frank Federico, B.S., R.Ph.; Marilyn Flack; Steven S. Fountain, M.D.; Suzanne Graham, R.N., Ph.D.; Martin J. Hatlie, Esq.; Jennifer Jackson, B.S.N., J.D.; Paul Kelley, CBET; Henri R. Manasse, Jr., Ph.D., Sc.D.; Jane McCaffrey, MHSA, DFASHRM; Mark W. Milner, R.N., MBA, CPHQ, FACHE; Jeanine Arden Ornt, J.D.; Grena Porto, R.N., M.S., ARM, CPHRM; Matthew Scanlon, M.D.; Ronni P. Solomon, J.D.; Dana Swenson, P.E., MBA 